\title{
Thalidomide may be an effective drug for Blau syndrome: a case report
}

\author{
Wei Wang $^{1 *}$, Wei Wang ${ }^{1 *}$, Lin-Qing Zhong ${ }^{1}$, Wen-Dao Li $^{1}$, Shi-Jing $\mathrm{Wu}^{2}$, Hong-Mei Song ${ }^{1}$ \\ ${ }^{1}$ Chinese Academy of Medical Sciences, Peking Union Medical College, Peking Union Medical College Hospital Pediatrics, Beijing, China; ${ }^{2}$ Chinese \\ Academy of Medical Sciences, Peking Union Medical College, Peking Union Medical College Hospital Ophthalmology, Beijing, China \\ Correspondence to: Hong-Mei Song. Peking Union Medical College Hospital (Dongdan Campus), Beijing 100730, China. \\ Email: songhm1021@hotmail.com.
}

\begin{abstract}
Blau syndrome (BS) is a monogenic autoinflammatory disease caused by mutations in nucleotide-binding oligomerization domain containing 2 (NOD2). BS is characterized by the clinical triad of granulomatous dermatitis, arthritis and recurrent uveitis. Due to the low incidence of BS and the lack of treatment studies with large samples, a specific treatment scheme has not been established. We report the case of a patient with BS that was uncontrollable with various immunosuppressive therapies but had a good response to thalidomide. She had the typical triad of rash, arthritis and uveitis. Gene sequencing indicated a NOD2 heterozygous missense variant (c.1759C > T, p.R587C), which has been reported as a pathogenic mutation. The BS diagnosis was confirmed. After treatment with methotrexate, an anti-tumour necrosis factor (TNF)- $\alpha$ inhibitor and corticosteroids, the patient's clinical symptoms and inflammatory indicators remained uncontrolled, and she experienced multiple side effects, such as hypertension and growth retardation attributed to prolonged corticosteroid use. After treatment with thalidomide, her condition was controlled without recurrence or side effects, and corticosteroids were stopped as soon as possible. This report suggests that thalidomide may be effective for BS treatment, but more research is needed to evaluate its long-term efficacy and side effects.
\end{abstract}

Keywords: Blau syndrome (BS); treatment; thalidomide; case report

Submitted Aug 10, 2021. Accepted for publication Oct 18, 2021.

doi: 10.21037/apm-21-2216

View this article at: https://dx.doi.org/10.21037/apm-21-2216

\section{Introduction}

Blau syndrome (BS, MIM \#186580) is a rare, dominantly inherited autoinflammatory disorder associated with gain-of-function mutations in the nucleotide-binding oligomerization domain containing 2 (NOD2) gene. BS is characterized by the clinical triad of granulomatous dermatitis, arthritis and recurrent uveitis (1). BS is a progressive disease that can lead to blindness (2); therefore, early treatment is necessary to reduce the incidence of sequelae. Due to the low incidence of BS and the lack of treatment studies with large samples, a specific treatment scheme has not been established. We report here the case of a Chinese girl with BS that was uncontrollable with various immunosuppressive therapies but had a good response to thalidomide. Therefore, we propose that thalidomide is a potential drug for refractory BS, and there are very few reports about this. We present the following case in accordance with the CARE reporting checklist (available at https://apm.amegroups.com/article/view/10.21037/apm$21-2216 / \mathrm{rc})$.

\section{Case presentation}

The patient, a 10-year-old Chinese girl, developed papules

\footnotetext{
* "Wei Wang ${ }^{1 *}$ " and "Wei Wang ${ }^{1 *}$ " on the list of authors are different authors. The first "Wei Wang ${ }^{1 *}$ " is named “王伟” in Chinese. The second “Wei Wang" ${ }^{1 *}$ ” is named “王薇” in Chinese.
} 

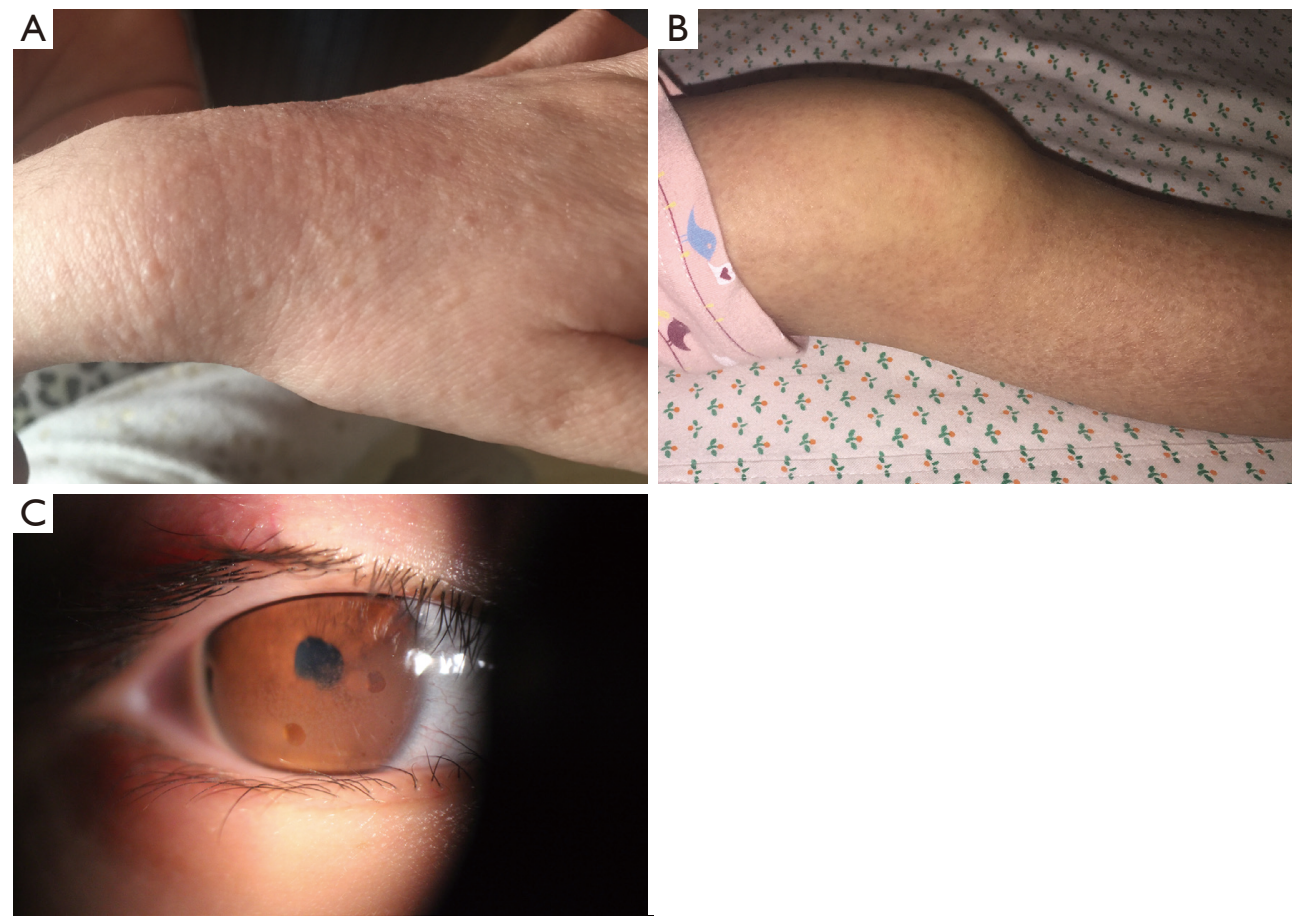

Figure 1 The manifestations of the patient: (A) a papular rash; (B) arthritis, with "boggy" synovitis of the knee; and (C) band keratopathy and partial posterior synechia of the iris as shown by slit-lamp photography of the eye. This image is published with the consent of the patient's parents.

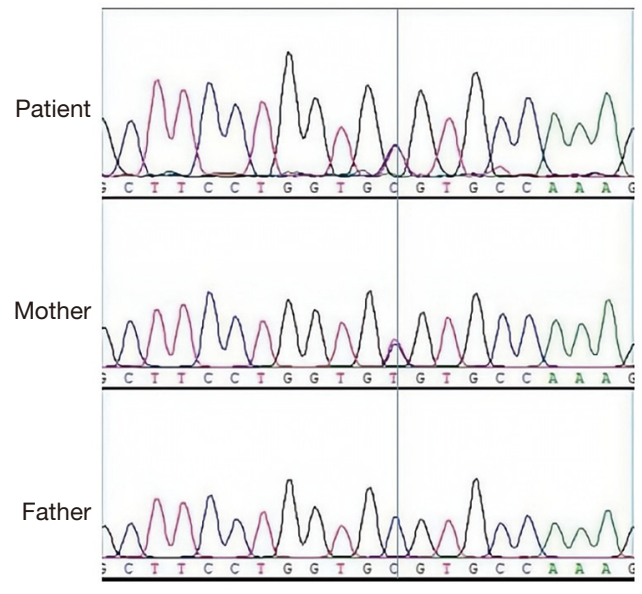

Figure 2 Diagram of Sanger sequencing results.

on the trunk and limbs at the age of 4 months (Figure 1A). A skin biopsy sample showed granulomatous dermatitis. At 3 years of age, she presented with a history of polyarthritis bilaterally involving the wrists, elbows, knees and ankles (Figure $1 \mathrm{~B}$ ). All the joints manifested boggy synovitis. Magnetic resonance imaging showed meningitis and oedema of the joints. She was diagnosed with juvenile idiopathic arthritis. She was healthy in the past, and a similar family history was denied. She was treated with methotrexate ( $2.5 \mathrm{mg}$ per week) with some benefit. The rash and arthritis were partially relieved. At the age of 4 years, she started complaining about increasing loss of eyesight. Ophthalmic examination revealed band keratopathy, partial posterior synechia of the iris of the eye and keratic precipitate similar to white jade, which is a manifestation of granulomatous panuveitis (Figure 1C). However, uvea biopsy was not performed. She was diagnosed with uveitis. At the age of 5 years, she experienced a high fever, and treatment with antibiotics was ineffective. Based on the presence of persistent arthritis, rash and uveitis, BS was suspected. To confirm the diagnosis, Sanger sequencing was performed. The patient had a maternally inherited c. $1759 \mathrm{C}>\mathrm{T}$, p. R587C mutation in the NOD2 gene (Figure 2), which has been reported as a pathogenic mutation. Therefore, our patient was diagnosed with BS. However, the mother denied a similar medical history.

Her clinical symptoms had several recurrences, and her erythrocyte sedimentation rate $(\mathrm{ESR}, 80 \mathrm{~mm} / \mathrm{h}$ at 


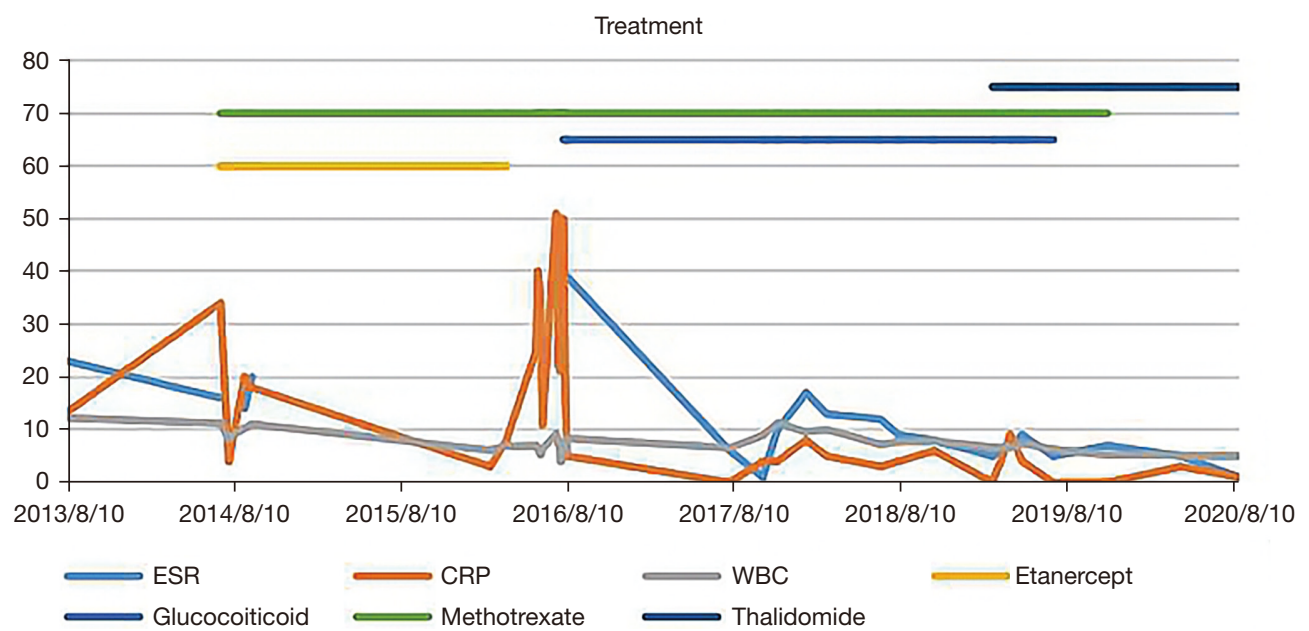

Figure 3 Clinical course of the patient. Thalidomide treatment was started at a dosage of $1 \mathrm{mg} / \mathrm{kg} / \mathrm{day}(25 \mathrm{mg} / \mathrm{d})$, beginning in February 2019. The patient's condition remained stable for 24 months despite thalidomide monotherapy. ESR, erythrocyte sedimentation rate; CRP, C-reactive protein; WBC, white blood cell.

age 6 years) and C-reactive protein (CRP; $50 \mathrm{mg} / \mathrm{L}$ ) increased discontinuously over the next 5 years despite the various immunosuppressive therapies attempted, such as prednisolone (15 $\mathrm{mg}$ per day), methotrexate $(2.5 \mathrm{mg}$ per week), and etanercept ( $0.8 \mathrm{mg}$ per kilogram per week) (Figure 3). During the process, we tried to change etanercept to infliximab $(60 \mathrm{mg})$, but after the second treatment with infliximab, the patient's fever worsened, which was considered an adverse reaction to infliximab. The long-term use of glucocorticoids caused severe side effects, such as refractory hypertension and growth retardation [at 9 years, height $125 \mathrm{~cm}$ (P3-P10), weight $24 \mathrm{~kg}(\mathrm{P} 10-\mathrm{P} 25)$ ], even though the dose of glucocorticoids was reduced to $2.5 \mathrm{mg}$ per day. She was treated with thalidomide $(25 \mathrm{mg} / \mathrm{d})$ at the age of 9 years at a dosage of $1 \mathrm{mg} / \mathrm{kg} /$ day $(25 \mathrm{mg} / \mathrm{d})$. Glucocorticoids and methotrexate were stopped quickly due to severe side effects and long-term application. Her rash and arthritis were in remission, and her inflammatory markers completely returned to the normal range for more than one year despite treatment with a single drug. Neither the results of eye examinations nor visual acuity deteriorated. Her height, weight and blood pressure gradually returned to the normal range of children the same age [at 10 years, height $137 \mathrm{~cm}$ (P25-P50), weight $32 \mathrm{~kg}$ (P50)]. Our patient was monitored with 3 monthly clinical examinations, and no side effects, such as peripheral neuropathy or sleepiness, were documented throughout the treatment with thalidomide.

All procedures performed in this study were in accordance with the ethical standards of the institutional and/or national research committee(s) and with the guidelines of the Declaration of Helsinki (as revised in 2013). Written informed consent was obtained from the parents of the patient for the publication of this case report and accompanying images. A copy of the written consent is available for review by the editorial office of this journal.

\section{Patient perspective}

The patient's parents were grateful for the care she received and felt fortunate that she was alive. The activity levels, appetite and growth of the patient returned to normal. Thalidomide was very beneficial in this patient. The quality of life of the patient was improved.

\section{Discussion}

We report the case of a patient with BS that was uncontrollable with various immunosuppressive therapies but had a good response to thalidomide. Thalidomide can inhibit the inflammatory response and improve clinical symptoms in patients with BS, with fewer adverse reactions. We found that thalidomide has potential in the treatment of refractory BS. However, our case report involves only one patient, and the follow-up was not long enough to provide meaningful statistical results. More research is needed to verify our results.

The diagnosis of BS is difficult to make according to the clinical manifestations. Some patients are wrongly diagnosed with systemic juvenile idiopathic arthritis (3). 
Therefore, genetic testing is necessary for the diagnosis of BS. Our patient had granulomatous dermatitis, arthritis and recurrent uveitis. Gene sequencing showed a NOD2 missense variant (c.1759C > T; p.R587C) that was inherited from her mother, which has been reported in previous families $(2,3)$. According to previous reports, p.R587C is associated with a relatively high incidence of fever, in addition to the typical triad of BS. The manifestations of our patient were the same as those reported in the literature. However, the mother of the patient had no clinical manifestations. In 2016, Harada et al. (4) reported the case of a child with the E383K pathogenic mutation in NOD2 inherited from the father, but the father had no clinical symptoms. Therefore, incomplete penetrance may be a characteristic of the disease.

There is specific treatment scheme of BS. The protein encoded by NOD2 is an intracellular bacterial sensor protein. This protein recognizes the oligomerization of muramyl dipeptide (MDP) and interacts with receptor-interacting serine/threonine protein kinase 2 (RIP2) to activate RIP2 phosphorylation, leading to the activation of NF-kappa B and inducing the production of proinflammatory cytokines, chemokines and adhesion molecules (5). The plasma levels of interleukin (IL)-1 $\beta$, IL-6 and anti-tumour necrosis factor (TNF)- $\alpha$ in patients with BS have been reported to be significantly higher than those in healthy controls (3). According to small-sample studies, high-dose glucocorticoids can be used to control symptoms in the acute phase of BS, and low-dose glucocorticoids can help to control uveitis and joint symptoms in stable periods. Immunosuppressants such as methotrexate, azathioprine, cyclosporine and mycophenolate mofetil can be added to the treatment of patients with BS refractory to glucocorticoid therapy (6). Therefore, TNF- $\alpha$ inhibitors (7) or IL-1 inhibitors (3) combined with corticosteroids and/or other immunosuppressants have been used to treat BS. However, there are some reports indicating that these biologics may be ineffective (8).

Thalidomide was mainly used for sedation and as an antiemetic in the 1950s (9). At present, thalidomide can be used in the treatment of rheumatoid arthritis (10), Behcet's disease (11), inflammatory bowel disease (12) and so on. Studies have found that thalidomide has a strong immunomodulatory effect. Thalidomide can inhibit the synthesis of TNF- $\alpha$ (13), promote the degradation of TNF- $\alpha$ mRNA (14) and block the activation of NF-kappa B by inhibiting the activity of $\mathrm{I}-\kappa \mathrm{B}$ kinase (IKBK) in the
TNF- $\alpha /$ NF-kappa B signalling pathway (15). Etanercept is a biologic fusion protein in the TNF blocker class of medications that binds both TNF-alpha and TNF-beta to inhibit the inflammatory response in joints and skin, which is characteristic of these autoimmune disorders. Thalidomide can inhibit the NF-kappa B pathway via an upstream kinase; therefore, thalidomide may inhibit other inflammatory and immunoregulatory molecules in addition to TNF-alpha. This possibility may explain why thalidomide is more effective than etanercept. In 2010, Japanese scholars found that thalidomide dramatically improved the symptoms of BS. In that study, thalidomide downregulated NF-kappa B signalling by inhibiting IKK (16). The condition of our patient with BS was also controlled after treatment with thalidomide. Therefore, thalidomide may effectively inhibit severe inflammatory reactions in patients with $N O D 2$-related diseases.

Our patient experienced no side effects throughout treatment with thalidomide. Peripheral neuropathy is a common and severe side effect of thalidomide treatment, presenting as a sensory, painful neuropathy. The prevalence ranges from $1 \%$ to $70 \%(17,18)$. The risk factors for thalidomide neuropathy include the daily dosage, duration of drug exposure, and cumulative dosage. A prospective study identified the daily dosage as the main risk factor and found that the risk of neuropathy seems to be negligible for dosages less than $25 \mathrm{mg}$ per day, regardless of the duration of therapy (19). However, another study in a group of children showed that long-term application for $>10$ months and cumulative dosages of $>20 \mathrm{~g}$ of thalidomide were likely to increase the risk of peripheral neuropathy (20). Our patient was monitored with 3 monthly clinical examinations without neurologic examination, and she had no neuropathic symptoms, weakness or sensory loss 24 months after treatment with a dosage of $25 \mathrm{mg}$ per day after a cumulative dosage of $18 \mathrm{~g}$ of thalidomide. Therefore, the daily dosage of $25 \mathrm{mg}$ is probably safe. Longer follow-up is needed to monitor the side effects of thalidomide.

In summary, BS is characterized by granulomatous dermatitis, arthritis and recurrent uveitis. Thalidomide can inhibit the inflammatory response and improve clinical symptoms in patients with BS, with few adverse reactions. For patients with refractory BS, thalidomide use can be attempted, but at present, studies about the treatment of BS with thalidomide are few, and more research is needed to evaluate the efficacy and side effects of thalidomide. 


\section{Acknowledgments}

Funding: This work is supported by the Beijing Natural Science Foundation (L202050); the Chinese Academy of Medical Sciences Innovation Fund for Medical Sciences (CIFMS) (2016-I2 M-1-008); the Public Welfare Scientific Research Project of China (201402012); and the National Key Research and Development Program of China (2016YFC0901500).

\section{Footnote}

Reporting Checklist: The authors have completed the CARE reporting checklist. Available at https://apm.amegroups. com/article/view/10.21037/apm-21-2216/rc

Conflicts of Interest: All authors have completed the ICMJE uniform disclosure form (available at https://apm. amegroups.com/article/view/10.21037/apm-21-2216/coif). The authors have no conflicts of interest to declare.

Ethical Statement: The authors are accountable for all aspects of the work in ensuring that questions related to the accuracy or integrity of any part of the work are appropriately investigated and resolved. All procedures performed in this study were in accordance with the ethical standards of the institutional and/or national research committee(s) and with the guidelines of the Declaration of Helsinki (as revised in 2013). Written informed consent was obtained from the parents of the patient for the publication of this case report and accompanying images. A copy of the written consent is available for review by the editorial office of this journal.

Open Access Statement: This is an Open Access article distributed in accordance with the Creative Commons Attribution-NonCommercial-NoDerivs 4.0 International License (CC BY-NC-ND 4.0), which permits the noncommercial replication and distribution of the article with the strict proviso that no changes or edits are made and the original work is properly cited (including links to both the formal publication through the relevant DOI and the license). See: https://creativecommons.org/licenses/by-nc-nd/4.0/.

\section{References}

1. Wouters $\mathrm{CH}$, Maes A, Foley KP, et al. Blau syndrome, the prototypic auto-inflammatory granulomatous disease.
Pediatr Rheumatol Online J 2014;12:33.

2. Matsuda T, Kambe N, Ueki Y, et al. Clinical characteristics and treatment of 50 cases of Blau syndrome in Japan confirmed by genetic analysis of the NOD2 mutation. Ann Rheum Dis 2020;79:1492-9.

3. Aróstegui JI, Arnal C, Merino R, et al. NOD2 geneassociated pediatric granulomatous arthritis: clinical diversity, novel and recurrent mutations, and evidence of clinical improvement with interleukin-1 blockade in a Spanish cohort. Arthritis Rheum 2007;56:3805-13.

4. Harada J, Nakajima T, Kanazawa N. A Case of Blau Syndrome with NOD2 E383K Mutation. Pediatr Dermatol 2016;33:e385-7.

5. Yao Q. Nucleotide-binding oligomerization domain containing 2: structure, function, and diseases. Semin Arthritis Rheum 2013;43:125-30.

6. Simonini G, Xu Z, Caputo R, et al. Clinical and transcriptional response to the long-acting interleukin-1 blocker canakinumab in Blau syndrome-related uveitis. Arthritis Rheum 2013;65:513-8.

7. Chen J, Luo Y, Zhao M, et al. Effective treatment of TNF $\alpha$ inhibitors in Chinese patients with Blau syndrome. Arthritis Res Ther 2019;21:236.

8. PaÇ Kisaarslan A, SÖzerİ B, Şahİn N, et al. Blau Syndrome and Early-Onset Sarcoidosis: A Six Case Series and Review of the Literature. Arch Rheumatol 2019;35:117-27.

9. McBride WG. Thalidomide and congenital abnormalities. Lancet 1961;2:1358.

10. Lehman TJ, Schechter SJ, Sundel RP, et al. Thalidomide for severe systemic onset juvenile rheumatoid arthritis: A multicenter study. J Pediatr 2004;145:856-7.

11. Letsinger JA, McCarty MA, Jorizzo JL. Complex aphthosis: a large case series with evaluation algorithm and therapeutic ladder from topicals to thalidomide. J Am Acad Dermatol 2005;52:500-8.

12. Yang C, Singh P, Singh H, et al. Systematic review: thalidomide and thalidomide analogues for treatment of inflammatory bowel disease. Aliment Pharmacol Ther 2015;41:1079-93.

13. Majumder S, Sreedhara SR, Banerjee S, et al. TNF $\alpha$ signaling beholds thalidomide saga: a review of mechanistic role of TNF- $\alpha$ signaling under thalidomide. Curr Top Med Chem 2012;12:1456-67.

14. Tavares JL, Wangoo A, Dilworth P, et al. Thalidomide reduces tumour necrosis factor-alpha production by human alveolar macrophages. Respir Med 1997;91:31-9.

15. Keifer JA, Guttridge DC, Ashburner BP, et al. Inhibition of 
NF-kappa B activity by thalidomide through suppression of IkappaB kinase activity. J Biol Chem 2001;276:22382-7.

16. Yasui K, Yashiro M, Tsuge M, et al. Thalidomide dramatically improves the symptoms of early-onset sarcoidosis/Blau syndrome: its possible action and mechanism. Arthritis Rheum 2010;62:250-7.

17. Molloy FM, Floeter MK, Syed NA, et al. Thalidomide neuropathy in patients treated for metastatic prostate cancer. Muscle Nerve 2001;24:1050-7.

18. Briani C, Zara G, Rondinone R, et al. Thalidomide

Cite this article as: Wang W, Wang W, Zhong LQ, Li WD, Wu SJ, Song HM. Thalidomide may be an effective drug for Blau syndrome: a case report. Ann Palliat Med 2022;11(7):25382543. doi: 10.21037/apm-21-2216 neurotoxicity: prospective study in patients with lupus erythematosus. Neurology 2004;62:2288-90.

19. Bastuji-Garin S, Ochonisky S, Bouche P, et al. Incidence and risk factors for thalidomide neuropathy: a prospective study of 135 dermatologic patients. J Invest Dermatol 2002;119:1020-6

20. Priolo T, Lamba LD, Giribaldi G, et al. Childhood thalidomide neuropathy: a clinical and neurophysiologic study. Pediatr Neurol 2008;38:196-9. 